\title{
Visual system integrity and cognition in early Huntington's disease
}

Robert Christian Wolf ${ }^{1^{*}}$, Fabio Sambataro ${ }^{2}$, Nenad Vasic ${ }^{3}$, Eva-Maria Baldas $^{4}$, Iris Ratheiser ${ }^{4}, \mathrm{G}$. Bernhard Landwehrmeyer ${ }^{4}$, Malte Sebastian Depping ${ }^{1}$, Philipp Arthur Thomann ${ }^{1}$, Reiner Sprengelmeyer ${ }^{5}$, Sigurd D. Süssmuth ${ }^{4}$, Michael Orth ${ }^{4}$

${ }^{1}$ Center for Psychosocial Medicine, Department of General Psychiatry, University of Heidelberg, Germany

${ }^{2}$ Brain Center for Motor and Social Cognition@UniPR, Istituto Italiano di Tecnologia, Parma, Italy

${ }^{3}$ Department of Psychiatry and Psychotherapy III, Ulm University, Germany

${ }^{4}$ Department of Neurology, Ulm University, Germany

${ }^{5}$ School of Psychology and Neuroscience, University of St Andrews, St Andrews, Scotland

* Corresponding author: Robert Christian Wolf, MD

University of Heidelberg, Center for Psychosocial Medicine, Department of General Psychiatry

Voßstraße 4, 69115 Heidelberg, Germany

e-mail: christian.wolf@med.uni-heidelberg.de

Tel: +49-6221-5636601; Fax: +49-6221-565327

Short title: Visual systems in early HD 


\section{Abstract}

Posterior cortical volume changes and abnormal visuomotor performance are present in patients with Huntington's disease (HD). However, it is unclear whether posterior cortical volume loss contributes to abnormal neural activity and whether activity changes predict cognitive dysfunction. Using magnetic resonance imaging (MRI), we investigated brain structure and visual network activity at rest in patients with early HD $(n=20)$ and healthy controls $(n=20)$. The symbol digit modalities test (SDMT) and subtests of the visual object and space perception (VOSP) battery were completed offline. For functional MRI data a group independent component analysis was used. Voxel-based morphometry was employed to assess regional brain atrophy, and "biological parametric mapping" analyses were included to investigate the impact of atrophy on neural activity. Patients showed significantly worse visuomotor and visual object performance than controls. Structural analyses confirmed occipitotemporal atrophy. In patients and controls, two spatiotemporally distinct visual systems were identified. Patients exhibited decreased activity in the left fusiform cortex and increased left cerebellar activity. These findings remained stable after correction for brain atrophy. Higher fusiform cortex activity was associated with better SDMT performance and with lower disease burden scores. These associations were absent when relating cerebellar function to task performance and disease burden. The results of this study suggest that regionally specific functional abnormalities of the visual system can account for worse visuomotor cognition in HD. However, occipital volume changes cannot sufficiently explain abnormal neural function in patients.

Keywords: functional magnetic resonance imaging; Huntington's disease; independent component analysis; neuropsychology; resting-state; occipital cortex 


\section{Introduction}

Huntington's disease $(H D)$ is a neurodegenerative CAG repeat movement disorder with psychiatric disturbances and cognitive deficits (Ross and Tabrizi 2011). The neuropathological hallmark of HD is a progressive loss of striatal neurons (Vonsattel et al. 1985). However, numerous studies have also shown widespread cortical neurodegeneration (Rosas et al. 2008; Gomez-Anson et al. 2009; Nopoulos et al. 2010; Aylward et al. 2011; Tabrizi et al. 2012; Tabrizi et al. 2013). In manifest HD, meta-analyses of brain structure have emphasized pronounced atrophy of inferior, lateral and medial prefrontal, premotor, sensorimotor, insular, temporoparietal and posterior cortical regions including the occipital cortex (Dogan et al. 2012; Lambrecq et al. 2013,). Similar to striatal volume loss, cortical volume loss in HD over time exceeds the physiological volume loss in healthy controls (Tabrizi et al. 2011; Tabrizi et al. 2012). Posterior cortical changes involving the occipital lobes have been reported frequently in HD. Occipital volume loss can be detected as far as fifteen years prior to motor onset (Nopoulos et al. 2010). Occipital changes progress over the preclinical period once approaching motor onset and are consistently found in symptomatic stages of the disease (Rosas et al. 2008; Dogan et al. 2012; Tabrizi et al. 2013). In line with these observations, in preclinical and manifest HD functional neuroimaging data point towards activity changes of the occipital cortex at rest and during active cognition (Kim et al. 2004; Chen et al. 2012; Mochel et al. 2012; Novak et al. 2012; Reetz et al. 2012).

Some cortical changes were able to predict cognitive dysfunction in HD patients. For instance, several reports have shown contributions of orbitofrontal, cingulate or insular volume loss to executive dysfunction and affective processing deficits (Peinemann et al. 2005; Henley et al. 2008; Hobbs et al. 2011; Ille et al. 2011). Only a few studies so far investigated an association of structural occipital cortical change with cognitive performance, suggesting that visual system function may be impaired in HD (Rosas et al. 2008; Scahill et al. 2013). This is important because abnormal visuomotor and visuospatial cognition are well-known in HD gene mutation carriers (Lemiere et al. 2004; Rosas et al. 2008; Beglinger et al. 2010; Say et al. 2011; Tabrizi et al. 2012; 
Tabrizi et al. 2013). In addition, tasks of visuomotor integration are among those with the highest rates of change over time (Tabrizi et al. 2013). It is possible that lower-level visual system dysfunction could contribute to the pattern of cognitive deficits found in HD. In particular, visual system neural activity, and its association with posterior cortical volume, may relate directly to visuospatial or visuomotor cognition in manifest HD.

To address these questions, we assessed visual cognition, and occipital lobe brain structure and brain function in early HD patients. To this end we used VBM-based structural MRI and a "restingstate" functional MRI (rs-fMRI) technique (Fox and Greicius 2010; van den Heuvel and Hulshoff Pol 2010). The rs-fMRI approach relies on the observation that several anatomically distinct neural networks show ongoing spontaneous modulations of the blood oxygen level-dependent signal in stimulation-free periods (Biswal et al. 1995; Fox et al. 2005; Fox et al. 2006). Neural systems at rest include sensorimotor, auditory, visual and several discrete lateral and medial prefrontal networks, which have been identified with high intra- and interindividual reliability across multiple independent data sets (Damoiseaux et al. 2006; Smith et al. 2009; Fox and Greicius 2010; Kelly et al. 2012). Importantly, the spatial distribution of neural systems at rest closely corresponds to neural activity patterns observed during active experimental stimulation. This suggests a close relationship between a distinct functional neural architecture at rest and specific perceptual, motor and higher-order cognition (Smith et al. 2009; Laird et al. 2012).

In this study, we used a multivariate statistical technique for group fMRI data analysis, i.e. Independent Component Analysis [ICA] (Calhoun et al. 2004; Calhoun et al. 2008). ICA has been successfully applied to decompose resting-state fMRI data into multiple spatiotemporally distinct neural systems (Calhoun et al. 2004; Calhoun et al. 2008) including networks associated with visual system function (Smith et al. 2009; Laird et al. 2012). We conducted two types of functional analyses: first, we investigated activity differences at rest between the groups without taking into account potential influences of regional brain atrophy. Since brain atrophy may affect brain activity at rest in manifest HD (Quarantelli et al. 2013) in a second step we integrated highresolution structural data with the functional data analyses, thus correcting for regional brain atrophy across the whole brain. We expected that HD patients would exhibit both structural and 
resting state functional abnormalities in posterior cortical regions related to visual system function. In addition, in HD patients we explored the relationship between neural activity at rest and visuomotor and visuospatial cognition. Here, we predicted that functional changes would be associated with participants' performance on tests of spatial cognition and visuomotor function.

\section{Materials and methods}

\subsection{Participants}

We analyzed data from 20 right-handed early HD patients (Shoulson stages 1 and 2) with a genetically confirmed CAG repeat expansion $(\geq 39)$ in the HTT gene. Patients were excluded from participation if they met DSM-IV-TR criteria of a current or life-time mental disorder or if they presented with a history of another neurological disease or a history of head trauma. Intact visual ability in patients was ensured by patient's self-reports which were confirmed by family members or other care-givers. Seven patients were not taking any medication, 5 were on anti-dyskinetics, 3 were on anti-depressants, 3 were on a combination of anti-dyskinetics and anti-depressants, and 2 were on memantine. The burden of HD pathology was estimated using a formula based on age and CAG repeat length ((CAGn-35,5) x age; (Penney et al. 1997).

Healthy controls included 20 right-handed participants matched for age and education. Participants with neurological disease or a psychiatric disorder according to DSM-IV-TR criteria were excluded. Patients and controls underwent a thorough neuropsychiatric examination including a semi-structured psychiatric interview to exclude axis-I and axis-II disorders according to DSM-IV-TR, the Hospital Anxiety and Depression (HADS) scale and the complete UHDRS (Huntington-Study-Group 1996). The "Multiple Choice Vocabulary Test" ("Mehrfach Wortschatz Intelligenztest"), Version B (MWT-B, (Lehrl et al. 1995) was used as a proxy for intelligence. All participants were recruited exclusively for rs-fMRI, i.e. no other experimental tasks were included in the scanning protocol. The study was approved by the local Ethics Committee (Ulm University, 
Germany). Written informed consent was obtained from all participants following a complete description of the study.

\subsection{Cognitive tasks}

We employed the symbol digit modalities test (SDMT, (Smith 1982) and three subtests from the Visual Object and Space Perception (VOSP) battery (Warrington and James 1991). Both tests are independent of language.

The SDMT allows an assessment of selective attention, visual scanning, and motor speed. SDMT performance has been consistently found to be impaired in manifest HD (e.g. Mahant et al. 2003; Lemiere et al. 2004; Rosas et al. 2008; Beglinger et al. 2010) and is one of the cognitive markers showing large effect sizes of progressive change (Tabrizi et al. 2013). Participants have to identify nine different symbols corresponding to the numbers $1-9$, and to write the correct number under the corresponding symbol. The participant is given 90 seconds each for completing the tests. A total score is calculated where higher scores indicate better performance.

The VOSP battery includes tests designed to probe specific and dissociable aspects of visual perception. Instructions and test requirements are kept simple to minimize the impact of other cognitive and motor skills. Behavioral deficits during VOSP performance are documented in both preclinical and manifest HD (Lawrence et al. 2000; Lemiere et al. 2004). For this study, three tests from the VOSP battery, all probing visual object function, were available for all participants. A shape detection test was used to determine participant's ability to complete the battery. During shape detection, participants were asked to identify the presence of an " $X$ " on twenty pattern sheets. One correct answer scores one point. A score below 15 points indicates an individual's inability to complete the battery. The silhouettes subtest requires the identification of 15 animal and 15 object silhouettes, each correct answer scoring one point. The object decision subtest consists of 20 sheets of four stimuli each. Only one stimulus depicts a known object, whereas the other three stimuli are distractors. Participants are asked to decide which of the stimuli represent the silhouette of the real object, each correct answer scoring one point. 


\subsection{MRI data acquisition}

\subsubsection{Functional data}

Functional data were acquired using a 3 T Magnetom ALLEGRA (Siemens, Erlangen, Germany) head MRI system located at the Department of Psychiatry and Psychotherapy III at Ulm University, Germany. MRI scanning was carried out in darkness, and the participants were explicitly instructed to relax without falling asleep, to keep their eyes closed, not to think about anything special and move as little as possible. Adherence to these instructions was verified by verbal contact before and immediately after the resting-state scan. T2*-weighted images were obtained using echo-planar imaging in an axial orientation (TR/TE=2000/30 ms, FoV $192 \mathrm{~mm}$, flip angle $80^{\circ}$, voxel size $3 \times 3 \times 3 \mathrm{~mm}$, 33 slices, slice thickness $3 \mathrm{~mm}$, gap $\left.1 \mathrm{~mm}\right)$. Within a session 180 whole-brain volumes were acquired. To account for MRI equilibration effects, prior to data processing the first 8 volumes of the time-series were discarded.

\subsubsection{Structural data}

High-resolution structural data were acquired using a 3 T Magnetom ALLEGRA (Siemens, Erlangen, Germany) head MRI system. The MRI parameters of the three-dimensional magnetization-prepared rapid gradient-echo (3D-MPRAGE) sequences were as follows: $\mathrm{TE}=$ $3.67 \mathrm{~ms} ; \mathrm{TR}=2200 \mathrm{~ms} ; \mathrm{TI}=1200 \mathrm{~ms} ; \mathrm{FOV}=282 \mathrm{~mm}$; slice plane=axial; slice thickness = 1.1 $\mathrm{mm}$; number of slices $=208$.

\subsection{MRI data analysis}

\subsubsection{FMRI: ICA and component selection}

Prior to ICA, data preprocessing was performed using the Statistical Parametric Mapping software package version 8 (SPM8, Wellcome Department of Cognitive Neurology, London) and MATLAB 7.3 (MathWorks, Natick, MA). Preprocessing included coregistration to the individual T1 image, correction for motion artifacts, spatial normalization and smoothing with a $9 \mathrm{~mm}$ full width at half maximum (FWHM) Gaussian kernel. To ensure that the groups do not significantly differ in 
terms of movement during the scan, we computed the mean relative Euclidean distance between movement parameters derived from the individual realignment files. A two-sample t-test performed on these measure did not reveal significant differences between the groups $(p=0.19)$. A spatial ICA was then computed on the entire data set using the "Group ICA for fMRI Toolbox" [GIFT; http://mialab.mrn.org/software/gift] (Correa et al. 2005). To increase the stability of the components, we used the ICASSO algorithm (Himberg et al. 2004) after repeating the ICA estimation 50 times with bootstrapping and permutation. The dimensionality of the functional data for each subject was reduced using Principal Component Analysis alternated with data concatenation across subjects, resulting in one aggregate mixing matrix for all subjects. An ICA decomposition using the Infomax algorithm was used to extract 21 Independent Components (ICs), consisting of group spatial maps and related time-courses. The 'minimum description length' criteria were used to estimate the order selection ( $\mathrm{Li}$ et al. 2007). The estimated ICs were used for a back reconstruction into individual ICs using the aggregate mixing matrix created during the dimensionality data reduction steps. The individual ICs consisting of individual spatial independent maps and time-courses were eventually spatially sorted using a spatial mask comprising the occipital cortex. This mask was created using occipital cortex landmarks (bilateral superior, middle, inferior occipital cortices) as defined by the Automatic Anatomical Labelling (AAL) atlas (Tzourio-Mazoyer et al. 2002). Two components of interest that showed a significant $(p<0.01)$ positive spatial correlation with this mask were chosen for $2^{\text {nd }}$ level within- and betweengroup analyses (see also Results section 3.3.1). Note that the three masks described above were only used to identify networks of interest and to select them for further analyses. Masks were neither used as "seeds" nor used to constrain $2^{\text {nd }}$ level within- and between-group comparisons on a specific set of brain regions.

\subsubsection{FMRI: Between-group comparisons uncorrected for brain atrophy}

After selecting components of interest, voxel weights of each subject's component were used as random effects variables and analyzed using SPM8. For within-group analyses, voxelwise onesample t-tests against the null hypothesis of zero magnitude were used to calculate within-group 
maps for each network ( $p<0.001$, uncorrected for height, $p<0.05$ corrected for spatial extent). On the $2^{\text {nd }}$ level, we compared spatial maps between controls and patients using two-sample-t-tests covaried for age and gender. To fully include those brain regions that were recruited by both groups we explicitly masked these between-group comparisons with a combined mask. This mask was created as follows: first, we computed one-sample t-tests per component and group. Subsequently, thresholded t-maps ( $p<0.05$, uncorrected) were binarized using the "AND" Boolean operator, thus producing spatial masks of the combined effect for each group. Significant between-group differences were assessed at $p<0.001$, uncorrected at the voxel level, $p<0.05$ corrected for spatial extent (Worsley et al. 1998).

\subsubsection{Structural MRI data analysis}

A voxel-based morphometry (VBM) analysis of structural data was performed to investigate changes of gray matter volume (GMV). Segmentation routines provided by a VBM-toolbox running within SPM8 (VBM8, http://dbm.neuro.uni-jena.de/vbm8) were used. The VBM8 algorithm relies on an adaptive "Maximum A Posterior" (MAP) technique which does not require a priori information about tissue probabilities (Rajapakse et al. 1997). Images were normalized and segmented into grey/white matter/cerebrospinal fluid, followed by partial volume estimation (Tohka et al. 2004), data denoising (Manjon et al. 2010), application of Markov Random Fields (Rajapakse et al. 1997) and DARTEL normalization (Ashburner 2007). Normalized GMV segments were smoothed using a Gaussian kernel of $9 \mathrm{~mm}$ FWHM. To test for GMV differences between the groups, a t-test was computed where age, gender and total intracranial volume were included as nuisance variables. We included an absolute threshold of 0.2 to prevent effects located at tissue border regions. Since we were interested in the spatial overlap between brain function and structure, we explicitly masked between-group comparisons using binarized maps (see previous section) combining effects per group and component at $p<0.05$, uncorrected. Significant between-group differences in brain volume were assessed at $p<0.001$, uncorrected at the voxel level, $p<0.05$ corrected for spatial extent (Worsley et al. 1998). 


\subsubsection{FMRI: Between-group comparisons corrected for brain atrophy}

To investigate neural activity differences controlled for brain atrophy, we used the Biological Parametric Mapping (BPM) toolbox (http://www.ansir.wfubmc.edu) (Casanova et al. 2007). Based on a voxel-wise use of the general linear model (GLM) this toolbox integrates information obtained by multiple imaging modalities, such as functional and structural MRI. Using BPM wholebrain structural data can be included as covariates. For each functional component of interest we investigated activity differences between the groups using two separate ANCOVA models, where individual GMV images (as preprocessed by the VBM8 segmentation routines) were included as covariates, together with age, gender and total intracranial volume. Significant between-group differences were assessed at $p<0.001$, uncorrected at the voxel level, $p<0.05$ corrected for spatial extent (Worsley et al. 1998).

\subsubsection{Stereotaxic coordinates and anatomical labelling}

Stereotaxic coordinates obtained from $2^{\text {nd }}$ level group analyses are reported as coordinates of cluster-maxima in MNI (Montreal Neurological Institute) space. Anatomical regions emerging from the between-group comparisons were labelled according Talairach Daemon (TD) labels (Lancaster et al. 2000) and the AAL atlas, implemented in the Wake Forest University (WFU) PickAtlas toolbox (http://fmri.wfubmc.edu/software/PickAtlas).

\subsection{Relationships between neural activity, cognitive performance and disease burden}

Correlation analyses within the HD patient group were calculated between indices of neural activity, cognitive performance (SDMT and VOSP) and disease burden $(p<0.05$, uncorrected for multiple comparisons). SDMT and VOSP subtest performance as well as disease burden were correlated with mean voxel weights from significant clusters emerging from BPM between-group comparisons (i.e. the fusiform cortex and the cerebellar clusters, as described below). Parameter extraction was performed using the MarsBar toolbox (Brett et al. 2002) and then processed offline using the Statistica software package (Version 6.0, StatSoft Inc., Tulsa, OK, U.S.A.). 


\section{$3 \quad$ Results}

\subsection{Participants}

Patients and controls were similar in age, gender distribution, years of education and IQ (Table 1). UHDRS motor, cognitive and functional capacity scores were all significantly worse in patients than in controls (table 1). HADS scores were similar in both groups.

\subsection{Cognitive performance}

SDMT performance was significantly worse in patients compared to controls (Table 2). VOSP shape detection performance did not differ between the groups. VOSP silhouettes and object decision subtests performance was significantly worse in patients compared to controls (Table 2).

\subsection{MRI results}

\subsubsection{FMRI: ICA and component selection}

Twentyone independent components were estimated, consisting of individual spatially independent maps and time-courses. In all participants, two components of interest were identified after spatial correlation (Figure 1). The two networks included voxels with a significant positive spatial correlation with the spatial distribution of the occipital sorting mask (see also section 2.4.1):

Network $1(r=0.20, p<0.001)$ predominantly comprised the bilateral calcarine fissure, middle and superior occipital cortices, cuneus, precuneus, lingual and fusiform cortices, superior and middle temporal regions, insula, superior, middle and medial frontal regions (see also Figure 1 and Table 1a, supplementary data for detailed stereotaxic coordinates and Z-scores).

Network $2(r=0.17, p<0.001)$ predominantly included bilateral cerebellar, inferior occipital, inferior parietal, medial frontal and left pre- and postcentral areas (see also Figure 1 and Table 1a, supplementary data for detailed stereotaxic coordinates and Z-scores). 


\subsubsection{FMRI: Between-group comparisons uncorrected for brain atrophy}

Within network 1, between-group analyses not covaried for brain atrophy revealed, in HD patients, decreased activity in the left fusiform cortex $(x=-34, y=-52, z=-16, Z=4.12, k=236$ voxels). Within network 2, patients showed increased left cerebellar activity compared to controls $(x=-22$, $y=-40, z=-22, Z=5.05, k=218)$; see also Figure 2 .

\subsubsection{Structural MRI results}

Patients had less GMV than controls in bilateral middle occipital cortices, bilateral cuneus and precuneus, bilateral lingual cortices, left superior temporal cortex and right insula (Figure 3; see Table 2 for detailed stereotaxic coordinates and Z-scores). There were no brain regions with higher GMV in HD patients compared to controls.

\subsubsection{FMRI: Between-group comparisons corrected for brain atrophy}

BPM analyses confirmed lower left fusiform cortex activity in patients compared to controls ( $\mathrm{X}=-$ 34, $y=-52, z=-16, Z=4.01, k=155)$. BPM also confirmed higher left cerebellar activity in patients $(x=-22, y=-40, z=-22, Z=4.86, k=202)$; see also Figure 2 .

3.4. Relationships between neural activity, cognitive performance and disease burden Significant correlations in the patient group were found for fusiform cortex activity and SDMT performance as well as for fusiform cortex activity and disease burden. Higher left fusiform activity was associated with better SDMT performance $(r=0.54, p=0.006)$. Higher left fusiform activity was also associated with a lower disease burden $(r=-0.45, p=0.023)$; see also Figure 2. Other significant relationships between brain activity and behavioral/clinical measures were not found. 


\section{Discussion}

In patients with early HD we investigated visual network activity at rest, the influence of regional atrophy on neural activity and the relationship between neural activity and cognition. Three main findings emerged: 1. In patients, left fusiform cortex activity was reduced and left cerebellar activity increased. 2. Volume loss in patients did not predict functional change. 3. Higher left fusiform activity was associated with better SDMT performance and with lower disease burden.

Cortical volume loss is a robust finding in patients with manifest HD and also in gene mutation carriers (see e.g. Peinemann et al. 2005; Henley et al. 2008; Hobbs et al. 2011; Ille et al. 2011; Tabrizi et al. 2011; Tabrizi et al. 2012). Here, we specifically confirmed occipital cortex atrophy in patients, as described previously (Rosas et al. 2008; Nopoulos et al. 2010; Tabrizi et al. 2012; Scahill et al. 2013; Tabrizi et al. 2013); see also Dogan et al. 2012 for a meta-analysis). We add to this in that we explored what these structural changes mean for visual system activity. Adding the functional dimension to structural findings is relevant since the relationship between behavior and neural activity may be closer than between brain structure and behavior. Moreover, recent functional neuroimaging data in manifest HD indicate that some functional changes, e.g. in the caudate nucleus or in cortical regions, can be explained by regional atrophy (Quarantelli et al. 2013). In line with previous studies (e.g. Damoiseaux et al. 2006; Smith et al. 2009; Laird et al. 2012) we identified two spatiotemporally distinct visual systems in HD patients and controls. We first performed between-group analyses without correcting for structural change. This revealed that HD patients had lower neural activity in the left fusiform cortex and higher activity in the left cerebellum. Activation differences in both brain regions survived whole-brain atrophy correction using BPM. This indicates that the activation differences in these two regions are not simply the consequence of brain volume loss. At the same time, volume loss in other regions was not accompanied by activation differences. In line with previous studies (Quarantelli et al. 2013; Werner et al. 2013) this suggests that, at least in the occipital lobes, regional atrophy does not necessarily give rise to abnormal neural activity at rest in manifest HD (Chen et al. 2012). This is remarkable given the widespread occipital cortical volume loss; only one region of the occipital 
lobe was found to be functionally abnormal in patients. We are, however, aware that we refer to data acquired at rest. Occipital lobe activity in task-based fMRI differed more widely suggesting a different structure-function relationship when the brain is challenged with a task rather than at rest (Kim et al. 2004; Novak et al. 2012) could show different patterns of between-group change. RsfMRI could therefore complement structural MRI and task-based data so that the structurefunction relationship at rest can be compared to the structure-function relationship when faced with a paradigm challenging visual system function.

We next explored the clinical and behavioral relevance of these findings. We therefore examined the association between atrophy-corrected neural activity changes with clinical and behavioral parameters. This revealed a significant association between fusiform cortex activity and both SDMT performance and disease burden: higher fusiform activity was associated with better SDMT performance and with lower disease burden scores. Several task-based fMRI studies in healthy volunteers have linked occipital cortical activity and SDMT performance (Forn et al. 2009; Usui et al. 2009; Forn et al. 2013). When varying task load in an SDMT paradigm ICA revealed that the functional connectivity of a left lateralized fusiform cortical network was modulated by task-demand as a function of increased information processing speed (Forn et al. 2013). There may be several distinct processes that rely on the integrity of fusiform cortical activity during SDMT performance. The SDMT taps into multiple visual, motor and cognitive domains, such as visual perception, visual attention and scanning, memory and motor function. Abnormal activity of the fusiform cortex alone is unlikely to explain impairments in all of these domains. Nevertheless, the extent of fusiform cortical activity is modulated by attentional demand (Hopfinger et al. 2001; Schwartz et al. 2005) and visual search processes (Donner et al. 2000; Hayakawa et al. 2006) both of which are required for successful SDMT completion. Fusiform cortex also contributes to the saccadic control that is needed for visual search processes (Fox et al. 1985; Morris and McCarthy 2007; Perneczky et al. 2011). Oculomotor deficits are well-documented in HD (see e.g. Hicks et al. 2008; Robert et al. 2009). Therefore, the relationship between fusiform cortex activity and SDMT performance in HD could be explained by oculomotor deficits. Patients also did worse than controls on VOSP silhouettes and object decision subtests. Object information processing 
critically relies on visual cortex function (Konen and Kastner 2008), but fusiform activity in patients did not predict VOSP subtests deficits. Tests of the VOSP battery focus on specific components of visual perception while minimizing an involvement of other cognitive skills (Warrington and James 1991). In contrast, the SDMT is attentionally more demanding and requires a higher degree of visual scanning, including oculomotor function, and visual information processing speed. Eventually, it is also noteworthy that SDMT performance is one of the cognitive markers showing large effect sizes of progressive change in manifest and in individuals carrying the HD gene mutation who are close to motor symptom onset (Tabrizi et al. 2013). Though derived from cross-sectional data, the association between fusiform cortical activity and disease burden scores are suggestive of a decline of fusiform cortex function over time. Loss of striatal volume, and its consequences for motor and cognitive performance, is a key finding in HD. The association between fusiform cortical activity and SDMT performance suggests that the fusiform cortex may follow a similar trajectory in HD. However, longitudinal data are needed to confirm this hypthesis.

Limitations of this study include the cross-sectional design, which did not allow testing for disease-related changes over time. It therefore remains unknown at this stage if, over time, visual system activity and structural loss follow similar temporal trajectories and to what extent progressive volume loss may then affect changes of neural activity. Also, thirteen patients received psychotropic drugs, including antidepressants, which may affect neural activity (McCabe and Mishor 2011; Posner et al. 2013). Even though effects of psychotropic medication on visual system function have not been consistently reported, and even though we observed similar neural dysfunction in unmedicated and medicated patients, we cannot fully rule out effects of medication., We also acknowledge that rs-fMRI is conducted within a poorly controlled experimental setting, and that unspecific factors such as changes in vigilance may affect brain activity in cortical and subcortical areas (Olbrich et al. 2009). However, vigilance regulation has only a moderate impact on visual attention, and these effects are not mirrored by task performance (Bekhtereva et al. 2013). Moreover, we observed regionally specific changes in regions, which may not be subject to vigilance fluctuations (Olbrich et al. 2009). Contributions of fusiform activity to SDMT performance have been previously described (Forn et al. 2009; Forn et 
al. 2013), so that we are confident that abnormal fusiform cortex activity in patients is related to important functional domains of the disease and not to transient processes which could confound resting-state scanning. Eventually, we are aware that brain activity during SDMT performance is not restricted to the occipital cortex. SDMT-related brain activation and functional connectivity essentially involves frontal and parietal regions thought to mediate other attentional and executive processes required during active task performance (Forn et al. 2009; Forn et al. 2013). Abnormalities in these systems may also contribute to poor SDMT performance and poor visual object recognition in patients. Investigating these relationships was however beyond the scope of this report.

In conclusion, we have confirmed previous findings indicative of widespread posterior cortical atrophy in early manifest HD. However, despite this volume loss changes of visual system activity at rest were limited to the left fusiform cortex. The majority of posterior cortical volume changes in early manifest HD therefore did not substantially affect visual system activity at rest. In contrast, left fusiform cortex activity changes survived atrophy correction, suggesting this is not simply the result of volume loss. The relationship between fusiform cortical activity changes and SDMT performance indicates behaviorally relevant contributions of extrastriate visual areas to cognition. In early HD activity changes of visual system function may not be limited to specific task-demands but may also extend to the resting brain. 


\section{$5 \quad$ Acknowledgements}

The authors would like to thank all participants and their families for their time and interest in this study, and Kathrin Brändle for excellent technical support. 


\section{$6 \quad$ References}

Ashburner, J., 2007. A fast diffeomorphic image registration algorithm. Neuroimage 38, 95-113.

Aylward, E.H., Nopoulos, P.C., Ross, C.A., Langbehn, D.R., Pierson, R.K., Mills, J.A., Johnson, H.J., Magnotta, V.A., Juhl, A.R., Paulsen, J.S., 2011. Longitudinal change in regional brain volumes in prodromal Huntington disease. J Neurol Neurosurg Psychiatry 82, 405-410.

Beglinger, L.J., Duff, K., Allison, J., Theriault, D., O'Rourke, J.J., Leserman, A., Paulsen, J.S., 2010. Cognitive change in patients with Huntington disease on the Repeatable Battery for the Assessment of Neuropsychological Status. J Clin Exp Neuropsychol 32, 573-578.

Bekhtereva, V., Sander, C., Forschack, N., Olbrich, S., Hegerl, U., Muller, M.M., 2013. Effects of EEGvigilance regulation patterns on early perceptual processes in human visual cortex. Clin Neurophysiol

Biswal, B., Yetkin, F.Z., Haughton, V.M., Hyde, J.S., 1995. Functional connectivity in the motor cortex of resting human brain using echo-planar MRI. Magnetic Resonance in Medicine 34, 537-541.

Brett, M., Anton, J.-L., Valabregue, R., Poline, J.B. (2002). Region of interest analysis using an SPM toolbox [abstract]. 8th International Conference on Functional Mapping of the Human Brain, Sendai, Japan, Available on CD-ROM in Neurolmage, Vol 16, No. 2.

Calhoun, V.D., Adali, T., Pekar, J.J., 2004. A method for comparing group fMRI data using independent component analysis: application to visual, motor and visuomotor tasks. Magn Reson Imaging 22, 11811191.

Calhoun, V.D., Kiehl, K.A., Pearlson, G.D., 2008. Modulation of temporally coherent brain networks estimated using ICA at rest and during cognitive tasks. Hum Brain Mapp 29, 828-838.

Casanova, R., Srikanth, R., Baer, A., Laurienti, P.J., Burdette, J.H., Hayasaka, S., Flowers, L., Wood, F., Maldjian, J.A., 2007. Biological parametric mapping: A statistical toolbox for multimodality brain image analysis. Neuroimage 34, 137-143.

Chen, J.J., Salat, D.H., Rosas, H.D., 2012. Complex relationships between cerebral blood flow and brain atrophy in early Huntington's disease. Neuroimage 59, 1043-1051.

Correa, N., Adali, T., Yi-Ou, L., Calhoun, V.D., 2005. Comparison of blind source separation algorithms for FMRI using a new Matlab toolbox: GIFT. Proc IEEE Int Conf Acoustics, Speech, Signal Processing 5, 401404.

Damoiseaux, J.S., Rombouts, S.A., Barkhof, F., Scheltens, P., Stam, C.J., Smith, S.M., Beckmann, C.F., 2006. Consistent resting-state networks across healthy subjects. Proc Natl Acad Sci U S A 103, 1384813853. 
Dogan, I., Eickhoff, S.B., Schulz, J.B., Shah, N.J., Laird, A.R., Fox, P.T., Reetz, K., 2012. Consistent Neurodegeneration and Its Association with Clinical Progression in Huntington's Disease: A CoordinateBased Meta-Analysis. Neurodegenerative Diseases 2012 Aug 23. [Epub ahead of print],

Donner, T., Kettermann, A., Diesch, E., Ostendorf, F., Villringer, A., Brandt, S.A., 2000. Involvement of the human frontal eye field and multiple parietal areas in covert visual selection during conjunction search. Eur J Neurosci 12, 3407-3414.

Forn, C., Belloch, V., Bustamante, J.C., Garbin, G., Parcet-Ibars, M.A., Sanjuan, A., Ventura, N., Avila, C., 2009. A symbol digit modalities test version suitable for functional MRI studies. Neurosci Lett 456, 11-14.

Forn, C., Ripolles, P., Cruz-Gomez, A.J., Belenguer, A., Gonzalez-Torre, J.A., Avila, C., 2013. Task-load manipulation in the Symbol Digit Modalities Test: an alternative measure of information processing speed. Brain Cogn 82, 152-160.

Fox, M.D., Corbetta, M., Snyder, A.Z., Vincent, J.L., Raichle, M.E., 2006. Spontaneous neuronal activity distinguishes human dorsal and ventral attention systems. Proc Natl Acad Sci U S A 103, 10046-10051.

Fox, M.D., Greicius, M., 2010. Clinical applications of resting state functional connectivity. Front Syst Neurosci 4, 19.

Fox, M.D., Snyder, A.Z., Vincent, J.L., Corbetta, M., Van Essen, D.C., Raichle, M.E., 2005. The human brain is intrinsically organized into dynamic, anticorrelated functional networks. Proc Natl Acad Sci U S A 102, 9673-9678.

Fox, P.T., Fox, J.M., Raichle, M.E., Burde, R.M., 1985. The role of cerebral cortex in the generation of voluntary saccades: a positron emission tomographic study. J Neurophysiol 54, 348-369.

Gomez-Anson, B., Alegret, M., Munoz, E., Monte, G.C., Alayrach, E., Sanchez, A., Boada, M., Tolosa, E., 2009. Prefrontal cortex volume reduction on MRI in preclinical Huntington's disease relates to visuomotor performance and CAG number. Parkinsonism and Related Disorders 15, 213-219.

Hayakawa, T., Fujimaki, N., Imaruoka, T., 2006. Temporal characteristics of neural activity related to target detection during visual search. Neuroimage 33, 296-306.

Henley, S.M., Wild, E.J., Hobbs, N.Z., Warren, J.D., Frost, C., Scahill, R.I., Ridgway, G.R., MacManus, D.G., Barker, R.A., Fox, N.C., Tabrizi, S.J., 2008. Defective emotion recognition in early HD is neuropsychologically and anatomically generic. Neuropsychologia 46, 2152-2160.

Hicks, S.L., Robert, M.P., Golding, C.V., Tabrizi, S.J., Kennard, C., 2008. Oculomotor deficits indicate the progression of Huntington's disease. Prog Brain Res 171, 555-558.

Himberg, J., Hyvarinen, A., Esposito, F., 2004. Validating the independent components of neuroimaging time series via clustering and visualization. Neuroimage 22, 1214-1222. 
Hobbs, N.Z., Pedrick, A.V., Say, M.J., Frost, C., Dar Santos, R., Coleman, A., Sturrock, A., Craufurd, D., Stout, J.C., Leavitt, B.R., Barnes, J., Tabrizi, S.J., Scahill, R.I., 2011. The structural involvement of the cingulate cortex in premanifest and early Huntington's disease. Mov Disord 26, 1684-1690.

Hopfinger, J.B., Woldorff, M.G., Fletcher, E.M., Mangun, G.R., 2001. Dissociating top-down attentional control from selective perception and action. Neuropsychologia 39, 1277-1291.

Huntington-Study-Group, 1996. Unified Huntington's Disease Rating Scale: reliability and consistency. Movement Disorders 11, 136-142.

Ille, R., Schafer, A., Scharmuller, W., Enzinger, C., Schoggl, H., Kapfhammer, H.P., Schienle, A., 2011. Emotion recognition and experience in Huntington disease: a voxel-based morphometry study. J Psychiatry Neurosci 36, 383-390.

Kelly, C., Biswal, B.B., Craddock, R.C., Castellanos, F.X., Milham, M.P., 2012. Characterizing variation in the functional connectome: promise and pitfalls. Trends Cogn Sci 16, 181-188.

Kim, J.S., Reading, S.A., Brashers-Krug, T., Calhoun, V.D., Ross, C.A., Pearlson, G.D., 2004. Functional MRI study of a serial reaction time task in Huntington's disease. Psychiatry Res 131, 23-30.

Konen, C.S., Kastner, S., 2008. Two hierarchically organized neural systems for object information in human visual cortex. Nat Neurosci 11, 224-231.

Laird, A.R., Fox, P.M., Eickhoff, S.B., Turner, J.A., Ray, K.L., McKay, D.R., Glahn, D.C., Beckmann, C.F., Smith, S.M., Fox, P.T., 2012. Behavioral interpretations of intrinsic connectivity networks. J Cogn Neurosci 23, 4022-4037.

Lambrecq, V., Langbour, N., Guehl, D., B., B., Burbaud, P., Rotgea, J.-Y., 2013,. Evolution of brain gray matter loss in Huntington's disease: a meta-analysis. European Journal of Neurology 20,

Lancaster, J.L., Woldorff, M.G., Parsons, L.M., Liotti, M., Freitas, C.S., Rainey, L., Kochunov, P.V., Nickerson, D., Mikiten, S.A., Fox, P.T., 2000. Automated Talairach atlas labels for functional brain mapping. Hum Brain Mapp 10, 120-131.

Lawrence, A.D., Watkins, L.H., Sahakian, B.J., Hodges, J.R., Robbins, T.W., 2000. Visual object and visuospatial cognition in Huntington's disease: implications for information processing in corticostriatal circuits. Brain 123 ( Pt 7), 1349-1364.

Lehrl, S., Triebig, G., Fischer, B., 1995. Multiple choice vocabulary test MWT as a valid and short test to estimate premorbid intelligence. Acta Neurol Scand 91, 335-345.

Lemiere, J., Decruyenaere, M., Evers-Kiebooms, G., Vandenbussche, E., Dom, R., 2004. Cognitive changes in patients with Huntington's disease (HD) and asymptomatic carriers of the HD mutation - a longitudinal follow-up study. J Neurol 251, 935-942. 
Li, Y.O., Adali, T., Calhoun, V.D., 2007. Estimating the number of independent components for functional magnetic resonance imaging data. Human Brain Mapping 28, 1251-1266.

Mahant, N., McCusker, E.A., Byth, K., Graham, S., 2003. Huntington's disease: clinical correlates of disability and progression. Neurology 61, 1085-1092.

Manjon, J.V., Coupe, P., Marti-Bonmati, L., Collins, D.L., Robles, M., 2010. Adaptive non-local means denoising of MR images with spatially varying noise levels. J Magn Reson Imaging 31, 192-203.

McCabe, C., Mishor, Z., 2011. Antidepressant medications reduce subcortical-cortical resting-state functional connectivity in healthy volunteers. Neuroimage 57, 1317-1323.

Mochel, F., N'Guyen, T.M., Deelchand, D., Rinaldi, D., Valabregue, R., Wary, C., Carlier, P.G., Durr, A., Henry, P.G., 2012. Abnormal response to cortical activation in early stages of Huntington disease. Mov Disord 27, 907-910.

Morris, J.P., McCarthy, G., 2007. Guided saccades modulate object and face-specific activity in the fusiform gyrus. Hum Brain Mapp 28, 691-702.

Nopoulos, P.C., Aylward, E.H., Ross, C.A., Johnson, H.J., Magnotta, V.A., Juhl, A.R., Pierson, R.K., Mills, J., Langbehn, D.R., Paulsen, J.S., 2010. Cerebral cortex structure in prodromal Huntington disease. Neurobiol Dis 40, 544-554.

Novak, M.J., Warren, J.D., Henley, S.M., Draganski, B., Frackowiak, R.S., Tabrizi, S.J., 2012. Altered brain mechanisms of emotion processing in pre-manifest Huntington's disease. Brain 135, 1165-1179.

Olbrich, S., Mulert, C., Karch, S., Trenner, M., Leicht, G., Pogarell, O., Hegerl, U., 2009. EEG-vigilance and BOLD effect during simultaneous EEG/fMRI measurement. Neuroimage 45, 319-332.

Peinemann, A., Schuller, S., Pohl, C., Jahn, T., Weindl, A., Kassubek, J., 2005. Executive dysfunction in early stages of Huntington's disease is associated with striatal and insular atrophy: a neuropsychological and voxel-based morphometric study. J Neurol Sci 239, 11-19.

Penney, J.B., Jr., Vonsattel, J.P., MacDonald, M.E., Gusella, J.F., Myers, R.H., 1997. CAG repeat number governs the development rate of pathology in Huntington's disease. Ann Neurol 41, 689-692.

Perneczky, R., Ghosh, B.C., Hughes, L., Carpenter, R.H., Barker, R.A., Rowe, J.B., 2011. Saccadic latency in Parkinson's disease correlates with executive function and brain atrophy, but not motor severity. Neurobiol Dis 43, 79-85.

Posner, J., Hellerstein, D.J., Gat, I., Mechling, A., Klahr, K., Wang, Z., McGrath, P.J., Stewart, J.W., Peterson, B., 2013. Antidepressants normalize the default mode network in patients with dysthymia. JAMA Psychiatry 70, 373-382. 
Quarantelli, M., Salvatore, E., Giorgio, S.M., Filla, A., Cervo, A., Russo, C.V., Cocozza, S., Massarelli, M., Brunetti, A., De Michele, G., 2013. Default-Mode Network Changes in Huntington's Disease: An Integrated MRI Study of Functional Connectivity and Morphometry. PLoS One 8, e72159.

Rajapakse, J.C., Giedd, J.N., Rapoport, J.L., 1997. Statistical approach to segmentation of single-channel cerebral MR images. IEEE Trans Med Imaging 16, 176-186.

Reetz, K., Romanzetti, S., Dogan, I., Sass, C., Werner, C.J., Schiefer, J., Schulz, J.B., Shah, N.J., 2012. Increased brain tissue sodium concentration in Huntington's Disease - a sodium imaging study at $4 \mathrm{~T}$. Neuroimage 63, 517-524.

Robert, M.P., Nachev, P.C., Hicks, S.L., Golding, C.V., Tabrizi, S.J., Kennard, C., 2009. Saccadometry of conditional rules in presymptomatic Huntington's disease. Ann N Y Acad Sci 1164, 444-450.

Rosas, H.D., Salat, D.H., Lee, S.Y., Zaleta, A.K., Pappu, V., Fischl, B., Greve, D., Hevelone, N., Hersch, S.M., 2008. Cerebral cortex and the clinical expression of Huntington's disease: complexity and heterogeneity. Brain 131, 1057-1068.

Ross, C.A., Tabrizi, S.J., 2011. Huntington's disease: from molecular pathogenesis to clinical treatment. Lancet Neurol 10, 83-98.

Say, M.J., Jones, R., Scahill, R.I., Dumas, E.M., Coleman, A., Santos, R.C., Justo, D., Campbell, J.C., Queller, S., Shores, E.A., Tabrizi, S.J., Stout, J.C., 2011. Visuomotor integration deficits precede clinical onset in Huntington's disease. Neuropsychologia 49, 264-270.

Scahill, R.I., Hobbs, N.Z., Say, M.J., Bechtel, N., Henley, S.M., Hyare, H., Langbehn, D.R., Jones, R., Leavitt, B.R., Roos, R.A., Durr, A., Johnson, H., Lehericy, S., Craufurd, D., Kennard, C., Hicks, S.L., Stout, J.C., Reilmann, R., Tabrizi, S.J., 2013. Clinical impairment in premanifest and early Huntington's disease is associated with regionally specific atrophy. Hum Brain Mapp 34, 519-529.

Schwartz, S., Vuilleumier, P., Hutton, C., Maravita, A., Dolan, R.J., Driver, J., 2005. Attentional load and sensory competition in human vision: modulation of $\mathrm{FMRI}$ responses by load at fixation during taskirrelevant stimulation in the peripheral visual field. Cereb Cortex 15, 770-786.

Smith, A. (1982). Symbol Digit Modalities Test. Los Angeles, Western Psychological Services.

Smith, S.M., Fox, P.T., Miller, K.L., Glahn, D.C., Fox, P.M., Mackay, C.E., Filippini, N., Watkins, K.E., Toro, R., Laird, A.R., Beckmann, C.F., 2009. Correspondence of the brain's functional architecture during activation and rest. Proc Natl Acad Sci U S A 106, 13040-13045.

Tabrizi, S.J., Reilmann, R., Roos, R.A., Durr, A., Leavitt, B., Owen, G., Jones, R., Johnson, H., Craufurd, D., Hicks, S.L., Kennard, C., Landwehrmeyer, B., Stout, J.C., Borowsky, B., Scahill, R.I., Frost, C., Langbehn, D.R., 2012. Potential endpoints for clinical trials in premanifest and early Huntington's disease in the TRACK-HD study: analysis of 24 month observational data. Lancet Neurology 11, 42-53. 
Tabrizi, S.J., Scahill, R.I., Durr, A., Roos, R.A., Leavitt, B.R., Jones, R., Landwehrmeyer, G.B., Fox, N.C., Johnson, H., Hicks, S.L., Kennard, C., Craufurd, D., Frost, C., Langbehn, D.R., Reilmann, R., Stout, J.C., 2011. Biological and clinical changes in premanifest and early stage Huntington's disease in the TRACKHD study: the 12-month longitudinal analysis. Lancet Neurology 10, 31-42.

Tabrizi, S.J., Scahill, R.I., Owen, G., Durr, A., Leavitt, B.R., Roos, R.A., Borowsky, B., Landwehrmeyer, B., Frost, C., Johnson, H., Craufurd, D., Reilmann, R., Stout, J.C., Langbehn, D.R., 2013. Predictors of phenotypic progression and disease onset in premanifest and early-stage Huntington's disease in the TRACK-HD study: analysis of 36-month observational data. Lancet Neurol 12, 637-649.

Tohka, J., Zijdenbos, A., Evans, A., 2004. Fast and robust parameter estimation for statistical partial volume models in brain MRI. Neuroimage 23, 84-97.

Tzourio-Mazoyer, N., Landeau, B., Papathanassiou, D., Crivello, F., Etard, O., Delcroix, N., Mazoyer, B., Joliot, M., 2002. Automated anatomical labeling of activations in SPM using a macroscopic anatomical parcellation of the MNI MRI single-subject brain. Neuroimage 15, 273-289.

Usui, N., Haji, T., Maruyama, M., Katsuyama, N., Uchida, S., Hozawa, A., Omori, K., Tsuji, I., Kawashima, R., Taira, M., 2009. Cortical areas related to performance of WAIS Digit Symbol Test: a functional imaging study. Neurosci Lett 463, 1-5.

van den Heuvel, M.P., Hulshoff Pol, H.E., 2010. Exploring the brain network: a review on resting-state fMRI functional connectivity. European Neuropsychopharmacology 20, 519-534.

Vonsattel, J.P., Myers, R.H., Stevens, T.J., 1985. Neuropathological classification of Huntington's disease. J Neuropathol Exp Neurol 44, 559-577.

Warrington, E.K., James, M. (1991). Visual Object and Space Perception Battery. Bury St Edmunds (UK), Thames Valley Test Company.

Werner, C.J., Dogan, I., Sass, C., Mirzazade, S., Schiefer, J., Shah, N.J., Schulz, J.B., Reetz, K., 2013. Altered resting-state connectivity in Huntington's Disease. Human Brain Mapping

Worsley, K.J., Cao, J., Paus, T., Petrides, M., Evans, A.C., 1998. Applications of random field theory to functional connectivity. Hum Brain Mapp 6, 364-367. 


\section{Table legends}

Table 1: Demographics and clinical scores for controls and HD patients.

UHDRS: Unified Huntington's disease rating scale; HADS: Hospital Anxiety and Depression Scale. n.a. indicates that these values were not available.

Table 2. Brain areas showing lower posterior cortical gray matter volume in early HD patients compared to healthy participants. Shown are results of the $2^{\text {nd }}$ level t-test, $p<0.001$ uncorrected at the voxel level, $p<0.05$ corrected for spatial extent. $k=$ no. of voxels within a cluster. 


\section{Figure legends}

Figure 1: Patterns of neural activity within two spatiotemporally distinct visual networks. Common to controls and HD, the figure displays independent components and their corresponding time courses, as identified by the group ICA. Red: visual network 1. Blue: visual network 2, see also section 3.3.1 for further details. The composite component maps were obtained using the graphical output function provided by the GIFT software (http://mialab.mrn.org/software/gift/index.html)

Figure 2: Occipitotemporal regions showing lower gray matter volume in early HD patients compared to healthy controls. Results of the $2^{\text {nd }}$ level t-test, $\mathrm{p}<0.001$ uncorrected for height, $\mathrm{p}<0.05$ corrected for spatial extent. The figure displays maps rendered onto the anatomical template implemented in SPM. The color bar represents Z-values.

Figure 3: Neural activity differences between early HD patients and controls. Results are derived from standard SPM and BPM between-group analyses, $p<0.001$ (uncorrected the voxel level), $p<0.05$ corrected for spatial extent. Shown are maps obtained using the graphical output function provided by the GIFT software (http://mialab.mrn.org/software/gift/index.html) and between-group differences, rendered onto the anatomical template implemented in SPM.

Figure 4: Correlation plots showing the relationship between measures of brain activity and behavioral/clinical measures in early HD patients (see section 3.4 for further details). Left: correlation between fusiform cortex activity and SDMT performance. Right: correlation between fusiform cortex activity and disease burden scores. 


\begin{tabular}{|c|c|c|c|c|c|}
\hline & \multicolumn{2}{|c|}{ controls $(n=20)$} & \multicolumn{2}{|c|}{ HD patients $(n=20)$} & \multirow[b]{2}{*}{$p$-value } \\
\hline & mean & sd & mean & sd & \\
\hline age (years) & 46.8 & 9.2 & 48.5 & 9.1 & 0.56 \\
\hline education (years) & 14.0 & 2.3 & 13.0 & 2.4 & 0.1 \\
\hline gender $(\mathrm{m} / \mathrm{f})$ & $13 / 7$ & & $14 / 6$ & & \\
\hline CAG repeat length & n.a. & & 43.4 & 1.9 & \\
\hline duration of illness (years) & n.a. & & 3.2 & 1.7 & \\
\hline disease burden & n.a. & & 374.6 & 69.4 & \\
\hline UHDRS motor & 1.1 & 1.2 & 25.1 & 11.0 & $0.000^{*}$ \\
\hline UHDRS functional capacity & 13 & 0 & 11.2 & 1.3 & $0.000^{*}$ \\
\hline IQ & 110.1 & 15.9 & 104.8 & 14.5 & 0.28 \\
\hline SDMT & 48.5 & 11.7 & 27.7 & 8.7 & $0.000^{*}$ \\
\hline VOSP shape detection & 19.5 & 0.6 & 19.8 & 0.4 & 0.24 \\
\hline VOSP silhouettes & 22.5 & 4.4 & 17.5 & 5.4 & $0.003^{*}$ \\
\hline VOSP object decision & 17.1 & 2.0 & 12.7 & 3.1 & $0.000^{*}$ \\
\hline HADS depression & 5.0 & 4.7 & 2.8 & 2.5 & 0.07 \\
\hline HADS anxiety & 5.4 & 3.4 & 6.3 & 4.3 & 0.47 \\
\hline
\end{tabular}

Table 1. Demographics and clinical scores for controls and early HD patients. UHDRS: Unified Huntington's disease rating scale; IQ: intelligence quotient; SDMT: Symbol digit modalities test; VOSP: Visual object and space perception battery; HADS: Hospital Anxiety and Depression Scale; n.a.: not applicable. * indicates significant differences between the groups. 


\begin{tabular}{lcccccc}
\hline anatomical region & $x$ & $y$ & $z$ & $Z$ & $k$ \\
\hline Left middle temporal gryus & -54 & -19 & -8 & 5.59 & 1188 \\
Left superior temporal gryus & -62 & -27 & -3 & 4.63 & \\
Right middle occipital gyrus & 23 & -87 & 13 & 5.47 & 6757 \\
Left cuneus & -14 & -90 & 4 & 4.53 & \\
Right cuneus & 27 & -85 & 31 & 4.18 & \\
Right lingual gyrus & 20 & -75 & -8 & 3.97 & \\
Right Insula & 35 & -25 & 10 & 5.06 & 491 \\
Left middle occipital gyrus & -27 & -79 & 19 & 4.54 & 678 \\
Left precuneus & -30 & -73 & 37 & 3.25 & \\
Right middle temporal gyrus & 65 & -27 & -15 & 4.38 & 424 \\
Left lingual gyrus & -27 & -75 & -17 & 4.18 & 375
\end{tabular}

Table

2.

Brain areas

showing lower gray matter volume in early HD patients compared to healthy participants. Shown are significant between-group differences derived from $2^{\text {nd }}$ between-group comparisons, $p<0.001$ uncorrected at the voxel level, $\mathrm{p}<0.05$ corrected for spatial extent. $\mathrm{k}=$ number of voxels within a cluster 


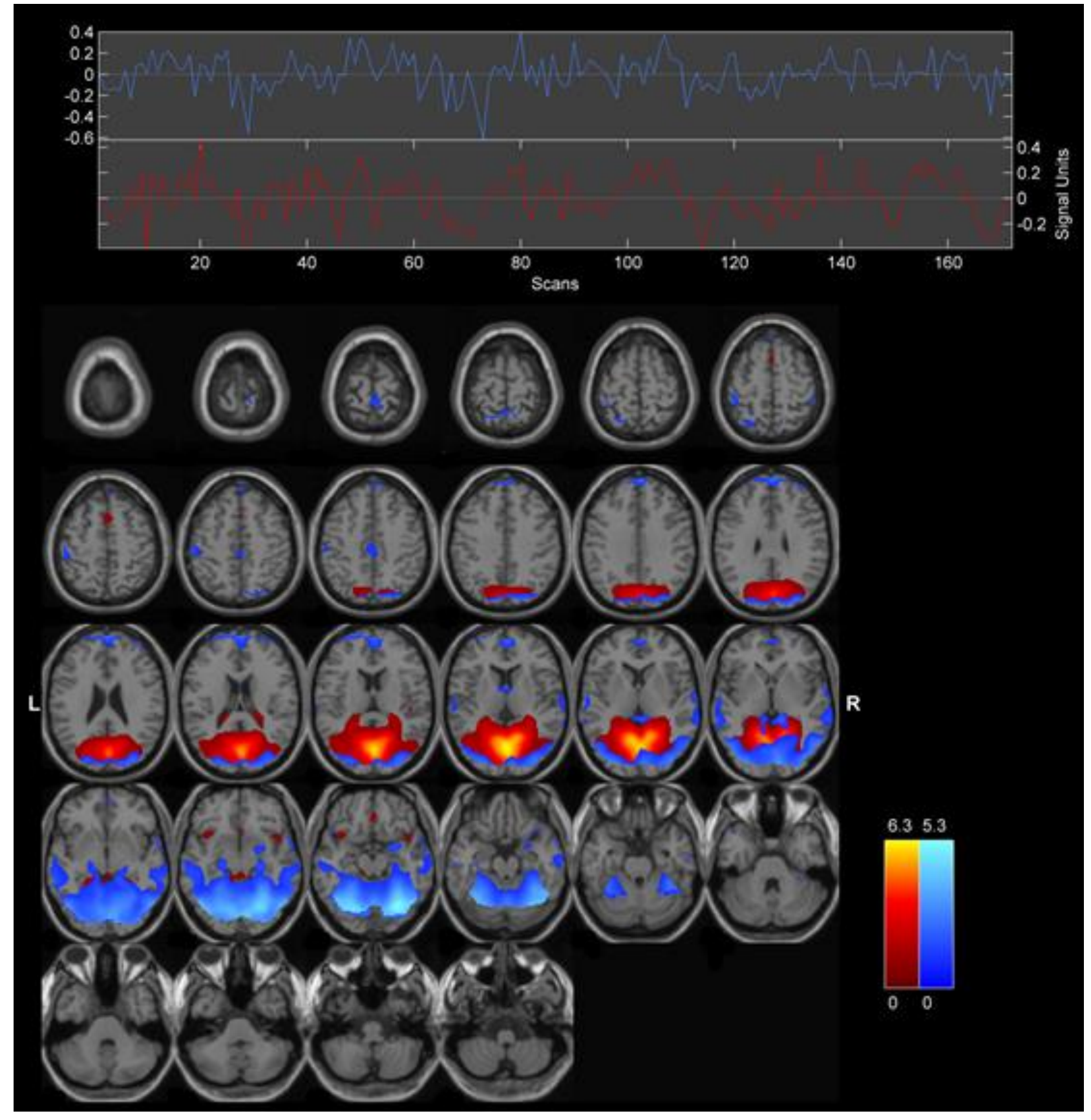

Figure 1 


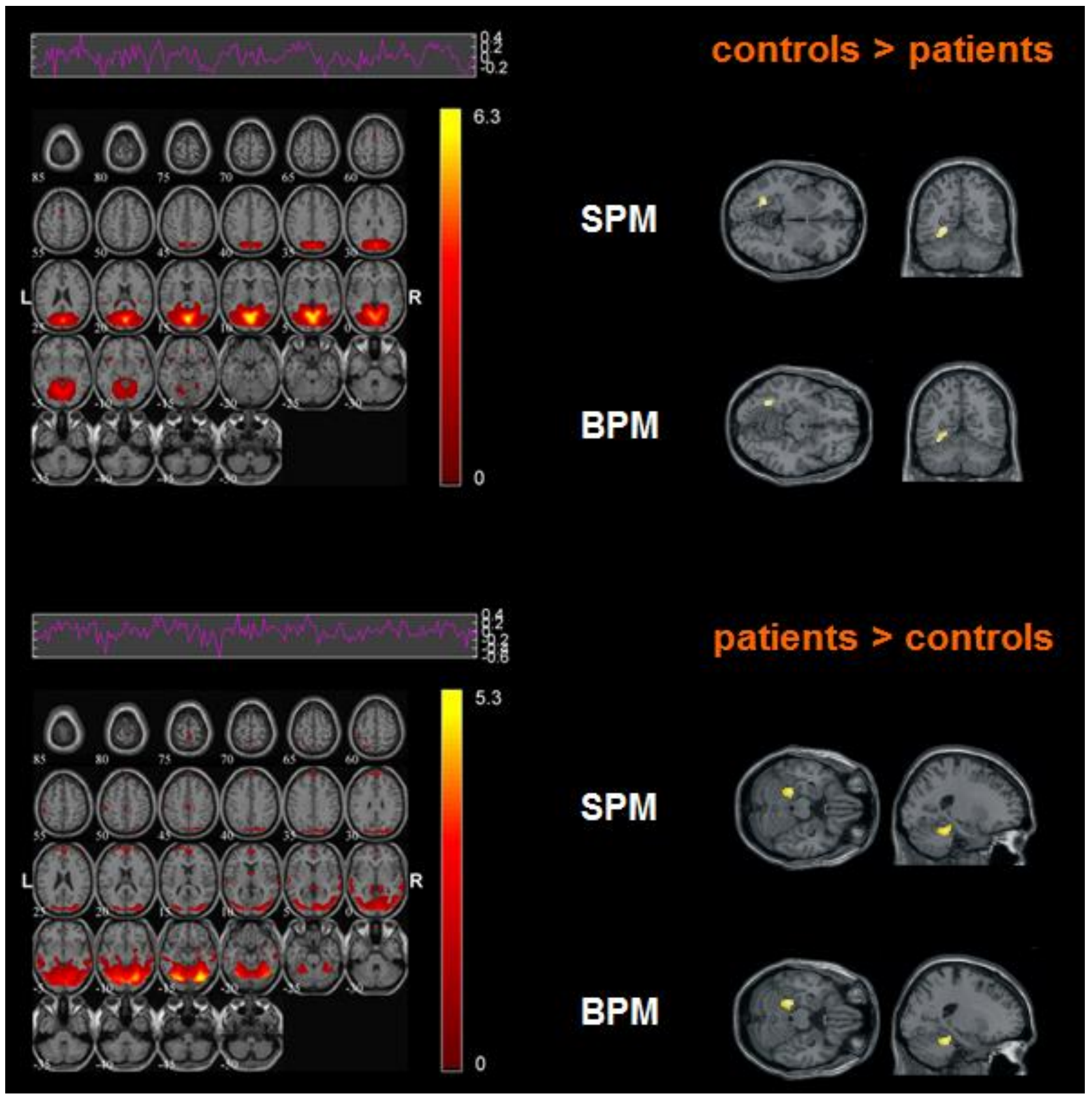

Figure 2 


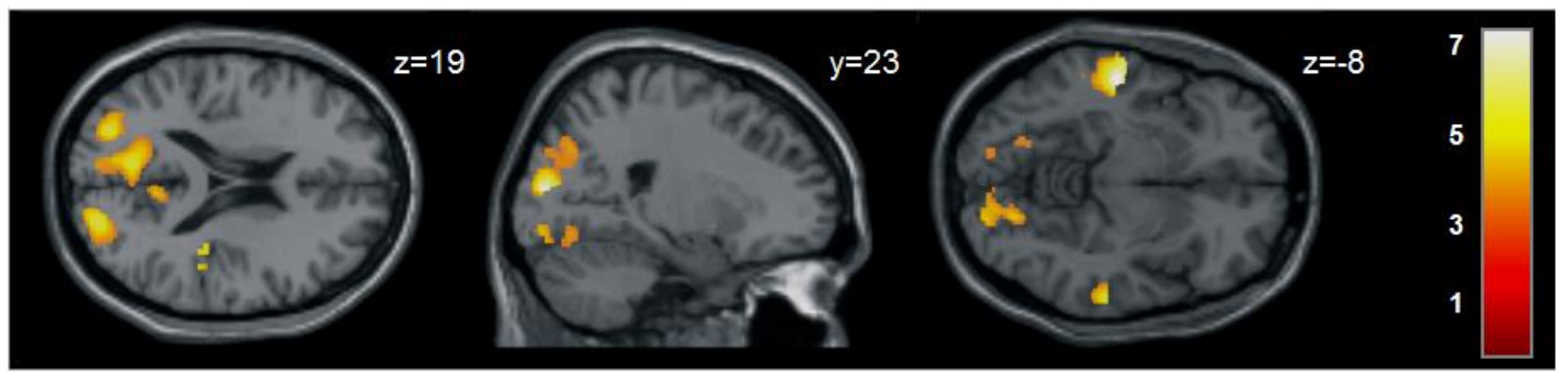

Figure 3
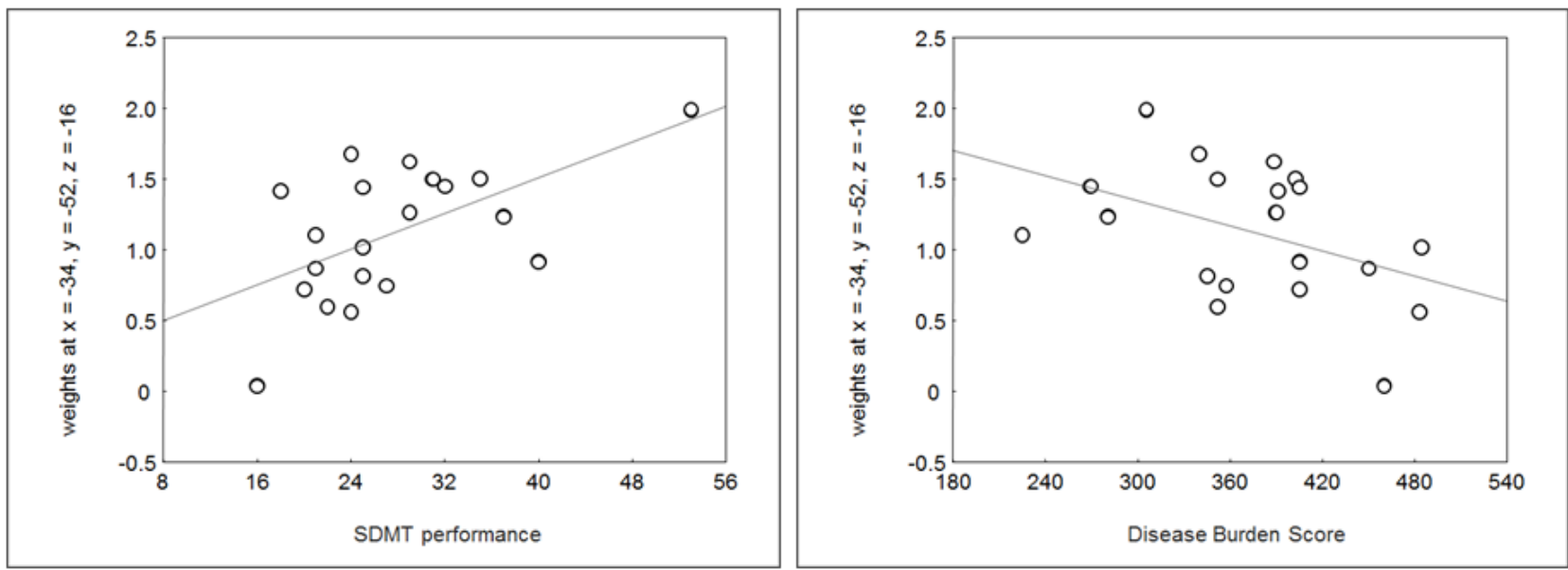

Figure 4 